\title{
MUTYH gene variants and breast cancer in a Dutch case-control study
}

\author{
Astrid A. Out • Marijke Wasielewski • Petra E. A. Huijts • Ivonne J. H. M. van Minderhout • \\ Jeanine J. Houwing-Duistermaat - Carli M. J. Tops • Maartje Nielsen • \\ Caroline Seynaeve · Juul T. Wijnen • Martijn H. Breuning • Christi J. van Asperen • \\ Mieke Schutte $\cdot$ Frederik J. Hes $\cdot$ Peter Devilee
}

Received: 22 November 2011 / Accepted: 16 January 2012/Published online: 2 February 2012

(C) The Author(s) 2012. This article is published with open access at Springerlink.com

\begin{abstract}
The MUTYH gene is involved in base excision repair. MUTYH mutations predispose to recessively inherited colorectal polyposis and cancer. Here, we evaluate an association with breast cancer (BC), following up our previous finding of an elevated BC frequency among Dutch bi-allelic MUTYH mutation carriers. A case-control study was performed comparing 1,469 incident BC patients (ORIGO cohort), 471 individuals displaying features suggesting a genetic predisposition for $\mathrm{BC}$, but without a detectable BRCA1 or BRCA2 mutation (BRCAx cohort), and 1,666 controls. First, for 303 consecutive patients
\end{abstract}

Marijke Wasielewski and Petra E. A. Huijts contributed equally to this work.

Electronic supplementary material The online version of this article (doi:10.1007/s10549-012-1965-0) contains supplementary material, which is available to authorized users.

A. A. Out · I. J. H. M. van Minderhout .

C. M. J. Tops - M. Nielsen - J. T. Wijnen .

M. H. Breuning - C. J. van Asperen - F. J. Hes

Department of Clinical Genetics, Leiden University Medical

Center, Leiden, The Netherlands

A. A. Out - P. E. A. Huijts - I. J. H. M. van Minderhout

J. T. Wijnen · P. Devilee $(\bowtie)$

Department of Human Genetics, Leiden University Medical

Center, P.O. Box 9600, 2300 RC Leiden, The Netherlands

e-mail: p.devilee@lumc.nl

Present Address:

A. A. Out

Department of Pathology, VU University Medical Center,

Amsterdam, The Netherlands

M. Wasielewski - M. Schutte

Department of Medical Oncology, Josephine Nefkens Institute,

Erasmus University Medical Centre, Rotterdam,

The Netherlands diagnosed before age 55 years and/or with multiple primary breast tumors, the MUTYH coding region and flanking introns were sequenced. The remaining subjects were genotyped for five coding variants, p.Tyr179Cys, p.Arg309Cys, p.Gly396Asp, p.Pro405Leu, and p.Ser515Phe, and four tagging SNPs, c.37-2487G $>$ T, p.Val22Met, c. $504+35 \mathrm{G}>\mathrm{A}$, and p.Gln338His. No bi-allelic pathogenic MUTYH mutations were identified. The pathogenic variant p.Gly396Asp and the variant of uncertain significance p.Arg309Cys occurred twice as frequently in BRCAx subjects as compared to incident BC patients and controls ( $p=0.13$ and $p=0.15$, respectively). The likely benign variant p.Val22Met occurred less frequently in patients from the incident $\mathrm{BC}(p=0.03)$ and BRCAx groups $(p=0.11)$, respectively, as compared to the controls. Minor allele genotypes of several MUTYH variants showed trends towards association with lobular BC histology. This

Present Address:

M. Wasielewski

Department of Genetics, University Medical Center Groningen, Groningen, The Netherlands

J. J. Houwing-Duistermaat

Department of Medical Statistics, Leiden University Medical

Center, Leiden, The Netherlands

C. Seynaeve

Department of Medical Oncology, Erasmus MC-Daniel den

Hoed Cancer Center, Rotterdam, The Netherlands

Present Address:

M. Schutte

Lorentz Center, Leiden, The Netherlands

P. Devilee

Department of Pathology, Leiden University Medical Center,

Leiden, The Netherlands 
extensive case-control study could not confirm previously reported associations of MUTYH variants with $\mathrm{BC}$, although it was too small to exclude subtle effects on BC susceptibility.

Keywords MUTYH - Breast cancer - BRCAx · Case-control study $\cdot$ Genotyping

\section{Introduction}

The MUTYH gene (muty homolog [Escherichia coli], MIM *604933) encodes a DNA glycosylase involved in base excision repair (BER). MUTYH, in cooperation with OGG1 and NUDT1, prevents G:C $>\mathrm{T}$ :A transversions, resulting from 8-oxo-G:A mispairs generated by oxidative damage. MUTYH germline mutations cause the recessively inherited phenotype: $M U T Y H$-associated polyposis (MAP, MIM \#608456), via somatic G:C > T:A mutations in APC and $K R A S$ [1-3]. The mono-allelic (heterozygous) pathogenic MUTYH mutation frequency is $1-2 \%$ in the population of European descent. Bi-allelic mutations (homozygous or compound heterozygous) are estimated to occur in 1:2,500-1:10,000. In Dutch MAP patients, three founder mutations (p.Tyr179Cys, p.Gly396Asp, and p.Pro405Leu) account for up to $90 \%$ of the pathogenic MUTYH mutations [4]. Bi-allelic MUTYH mutation carriers have a 28 -fold increased colorectal cancer (CRC) risk, while mono-allelic carriers have a moderately increased CRC risk (OR up to 1.34) [5].

Recently, two studies suggested a possible breast cancer (BC) risk for bi-allelic MUTYH mutation carriers. A significantly increased incidence $(4 / 22,18 \%)$ of $\mathrm{BC}$ was found among Dutch female bi-allelic MUTYH mutation carriers, as compared to the Dutch population [4]. Of these four BC patients, three were diagnosed before the age of 55 years and three had multiple breast tumors. In a next study, combining Dutch, German, and British MAP patients, 8/118 (6.8\%) females and 1/158 (0.6\%) males were diagnosed with $\mathrm{BC}$. The standardized incidence ratio (SIR) was significantly increased for male BC and also for female $\mathrm{BC}$, when taking into account multiple breast tumors per patient [6]. Furthermore, heterozygous MUTYH founder mutations were found to be significantly increased among index-patients with $\mathrm{BC}$ and/or CRC, from families with both BC and CRC $(6 / 138,4.3 \%)$, as compared to controls $(23 / 1192,1.9 \%)$, with exclusion of families with colorectal polyps [7], indicating a possible $\mathrm{BC}$ risk for MUTYH mutation heterozygotes.

Several independent findings support a possible role of MUTYH in BC. First, like MUTYH, many BC genes encode proteins involved in the DNA damage response, e.g., $B R C A 1 / 2$ and CHEK2. Second, oxidative DNA damage due to hormonal metabolism, including 8-oxoG, is believed to contribute to $\mathrm{BC}$ [8-12]. Third, somatic APC mutations have been described in a substantial proportion of breast carcinomas $(13 / 70,18 \%)$. Of the detected mutations, $5 / 15$ were substitutions, of which $4(80 \%)$ were G:C $>$ T:A [13]. Fourth, an increased frequency of mammary tumors was found in both $M U T Y H$ - and $A P C$-deficient mice compared to mice with an $A P C$ deficiency only [14]. Fifth, besides nuclear DNA damage, mitochondrial DNA (mtDNA) damage probably contributes to BC and MUTYH is abundantly expressed in mitochondria [15]. Finally, variants in the DNA mismatch repair (MMR) genes have been found to be weakly associated with BC [16].

The aim of this study was to extensively validate an association of MUTYH mutations with $\mathrm{BC}$, as suggested in three previous studies $[4,6,7]$. We first explored the MUTYH mutation spectrum in BC patients by sequencing the entire MUTYH coding region in 303 cases not selected for family history. We hypothesized that the MUTYH mutation spectrum could differ between $\mathrm{BC}$ and MAP patients. Next, we established the frequency of pathogenic MUTYH founder mutations in 1,469 incident BC patients and 1,666 controls. We also explored whether common MUTYH variants and haplotypes were associated with BC, by genotyping tagging single nucleotide polymorphisms (SNP). Finally, we investigated if non-BRCA1/BRCA2 familial BC patients (called BRCAx) have an increased MUTYH variant frequency, as compared to incident BC patients and controls. BRCAx patients might harbor so far undetermined high-risk gene variants and/or variants in multiple interacting high and low risk genes [17, 18]. This study reports the largest series of incident BC patients as reported to date and for the first time MUTYH mutation frequencies in BRCAx patients.

\section{Patients and methods}

\section{Patient samples}

Two BC patient groups were studied. The first group consisted of 1,518 incident BC patients, sampled for DNA examination in Rotterdam and Leiden between 1997 and 2008 ("ORIGO" cohort) [19]. Of this cohort, 1,469 remained after exclusion of patients with known $B R C A 1$ (33), BRCA2 (12), MLH1 (2), MSH2 (1), and PTEN (1) mutations. The second group was designated the "BRCAx" cohort, consisting of 471 unrelated subjects who underwent BRCA1/2 mutation screening at the Leiden University Medical Center (LUMC), The Netherlands, based on their family history and/or age at cancer diagnosis, but were not identified as BRCA1/2 mutation carriers. The 471 subjects included $376 \mathrm{BC}$ patients (of whom 
10 males), 29 ovarian cancer patients, and 7 patients with both breast and ovarian cancer. The remaining 59 subjects were tested for $B R C A 1 / 2$ mutations because of a strong family history of breast, ovarian or other $B R C A 1 / 2$-associated tumors [20]. As controls, 1,666 individuals were studied, originating from the South-Western part of the Netherlands, consisting of 165 partners of members from BC families, 254 female subjects screened for non-cancerrelated genetic diseases and 773 healthy female blood donors [7, 19]. Approval from the Medical Ethical Review Board of Leiden University Medical Centre and informed consent from all the individuals had been obtained (P06.060).

\section{Sequencing and genotyping}

Molecular genetic tests were performed on blood-derived DNA. Direct sequencing was performed for the coding region and exon/intron boundaries of $M U T Y H$ as described [4, 21]. Variants were annotated using NM_001128425.1 as reference sequence (www.lovd.nl/MUTYH, www.ncbi. nlm.nih.gov/genbank/) [22]. Genotyping of c.536A $>\mathrm{G}$ (p.Tyr179Cys), c.925C $>\mathrm{T}$ (p.Arg309Cys), c.1187G $>\mathrm{A}$ (p.Gly396Asp), c.1214C $>\mathrm{T}$ (p.Pro405Leu), and c.154 4C $>$ T (p.Ser515Phe) was done using Taqman ${ }^{\circledR}$ assays on an ABI 7500 instrument (Applied Biosystems, Inc., Foster City, CA, USA). The variants c.37-2487G $>$ T, c.64G $>A$ (p.Val22Met), c.504+35G $>$ A, and c.1014G $>$ C (p.Gln338His) were typed by Sequenom iPlex ${ }^{\circledR}$ (Sequenom, Inc., San Diego, CA, USA). Of the incident BC group, 214 and 291, of the 303 sequenced samples, were also analyzed by Taqman and Sequenom genotyping, respectively, with $100 \%$ concordancy of results. Primer sequences, PCR, Taqman, Sequenom, and sequence conditions are available on request. In silico analysis of potential deleterious effects of new MUTYH variants was performed with Alamut ${ }^{\circledR}$ Software (Interactive Biosoftware, Rouen, France).

\section{Statistics}

Chi-square tests were performed to test for differences in genotype frequencies between the study groups and the binary logistic regression analyses were used to estimate odds ratios, with the SPSS package version 16.0 (SPSS, Inc., Chicago, IL, USA). Associations of different phenotypical parameters with genotypes were analyzed by Chisquare tests, one-way analysis of variance (ANOVA), and binary logistic regression. Where applicable, $p$ values were calculated by a Fisher's exact test or estimated by a MonteCarlo simulation. A $p$ value below 0.05 was considered as statistically significant.

Power calculations showed that with 1,300 incident patients and 1,300 controls (a rounded estimate taking into account missing genotypes), and a heterozygote frequency of $2 \%$ in the controls, we had $80 \%$ power to detect heterozygote frequencies above $4 \%$ in the patients (OR 2.0) at $p<0.05$. With 450 BRCAx patients and 1,300 controls there is a power of $80 \%$ to detect a heterozygote frequency of $5 \%$ or higher (OR 2.6).

Unphased genotypes of the nine variants were loaded into Haploview software version 4.1 to test for associations of haplotypes and to test for Hardy-Weinberg equilibrium (HWE) [23].

\section{Results and discussion}

Sequencing of the MUTYH coding region

We first evaluated the spectrum of $M U T Y H$ variants by direct sequencing of the entire $M U T Y H$ coding region in 303 consecutively collected DNA samples from incident BC patients (ORIGO cohort). To enrich genetically susceptible cases, we selected cases with either a diagnosis before 55 years of age $(N=247)$ or with multiple primary BCs $(N=56$, Table 1$)$. Detected variants were compared to data from literature (www.lovd.nl/MUTYH, [1, 22, 24, 25] and dbSNP (build 133, www.ncbi.nlm.nih.gov/ projects/SNP). In the 303 patients, no bi-allelic pathogenic mutations were found. Of the three Dutch pathogenic founder mutations, only p.Gly396Asp was detected, in two heterozygous patients $(0.7 \%)$. The mutations, p.Tyr179Cys and p.Pro405Leu, were not found or any other known pathogenic mutations. This was lower compared to the total prevalence of heterozygous pathogenic MUTYH mutations in control populations of European descent (1-2\%) [26].

Eighteen patients $(5.9 \%)$ carried a variant of uncertain significance (VUS), of which 13 carried an intronic variant (without predicted RNA splice effect). We found three different coding VUS in five patients (c.56G $>$ A (p.Arg19Gln), c.312C >T (p.Tyr104Tyr), and three times c.925C > T (p.Arg309Cys)). The variant, p.Arg309Cys, has been described as a VUS in polyposis patients [6, 27]. Its effect was tested in one functional study, and showed normal glycosylase activity [28]. Three of the observed VUS were novel (c.56G $>$ A (p.Arg19Gln), c.1186+ $46 \mathrm{G}>\mathrm{A}$, and c. $1518+90 \mathrm{~A}>\mathrm{G}$ ), but in silico analyses showed no clear indications for pathogenicity (i.e., low Grantham scores, weak nucleotide and amino acid conservation and no predicted effects on RNA splicing were observed using Alamut). Common polymorphisms were found in similar frequencies as in populations of European descent. In the 56 patients with multiple primary breast tumors, the polymorphism c.64G $>$ A (p.Val22Met) was found about half as frequently, and the polymorphism c.1544C $>$ T (p.Ser515Phe) about twice as frequently, 
Table 1 MUTYH variants detected by direct sequencing in 303 incident BC patients diagnosed before the age of 55 years, of whom 56 had multiple primary breast tumors

\begin{tabular}{|c|c|c|c|c|c|c|}
\hline \multirow[t]{2}{*}{ Variant $^{\mathrm{a}}$} & \multicolumn{2}{|l|}{$N=303^{\mathrm{c}}$} & \multicolumn{2}{|c|}{$N=56$ with $>1$ breast tumor ${ }^{\mathrm{c}}$} & \multirow[t]{2}{*}{ Classification $^{\mathrm{d}}$} & \multirow[t]{2}{*}{ Rs number $^{\mathrm{d}}$} \\
\hline & No. het + hom & $\%$ MAF & No. het + hom & $\%$ MAF & & \\
\hline c. $-127 \mathrm{C}>\mathrm{T}$ & 20 & 3.3 & 4 & 3.6 & Polymorphism & rs3219466 \\
\hline c. $36+75 \mathrm{C}>\mathrm{G}$ & 2 & 0.3 & 0 & 0 & VUS & rs3219467 \\
\hline c.56G>A (p.Arg19Gln) & 1 & 0.2 & 0 & 0 & VUS, new & - \\
\hline c.64G $>$ A (p.Val22Met) & 29 & 4.8 & 2 & 1.8 & Polymorphism & rs3219484 \\
\hline c. $157+30 \mathrm{~A}>\mathrm{G}$ & 29 & 4.8 & 4 & 3.6 & Polymorphism & rs3219485 \\
\hline c. $312 C>T(p .=)$ & 1 & 0.2 & 0 & 0 & VUS & - \\
\hline c. $388+56 \mathrm{G}>\mathrm{A}$ & 2 & 0.3 & 0 & 0 & VUS & - \\
\hline c. $.504+35 \mathrm{G}>\mathrm{A}^{\mathrm{b}}$ & $58+5$ & 11.2 & $13+1$ & 13.4 & Polymorphism & rs3219487 \\
\hline c. $690+21 \mathrm{C}>\mathrm{A}$ & 4 & 0.7 & 0 & 0 & VUS & - \\
\hline c.925C > T (p.Arg309Cys) & 3 & 0.5 & 0 & 0 & VUS & - \\
\hline c. $998-27 \mathrm{G}>\mathrm{A}$ & 3 & 0.5 & 0 & 0 & VUS & - \\
\hline c.1014G >C (p.Gln338His) & $103+15$ & 21.9 & $20+4$ & 25.0 & Polymorphism & rs3219489 \\
\hline c. $1186+46 \mathrm{G}>\mathrm{A}$ & 1 & 0.2 & 1 & 0.9 & VUS, new & - \\
\hline c. $1187-27 \mathrm{C}>\mathrm{T}$ & 6 & 1.0 & 0 & 0 & Polymorphism & rs3219490 \\
\hline c.1187G>A (p.Gly396Asp) & 2 & 0.3 & 0 & 0 & Pathogenic & rs36053993 \\
\hline c. $1477-40 \mathrm{G}>\mathrm{C}^{\mathrm{b}}$ & $58+5$ & 11.2 & $13+1$ & 13.4 & Polymorphism & rs3219493 \\
\hline c. $1518+73 \mathrm{C}>\mathrm{T}$ & $0+1$ & 0.3 & 0 & 0 & Polymorphism & rs3219495 \\
\hline c. $1518+90 A>G$ & 1 & 0.2 & 1 & 0.9 & VUS, new & - \\
\hline c. $1544 \mathrm{C}>\mathrm{T}$ (p.Ser515Phe) & 10 & 1.7 & 5 & 4.5 & Polymorphism & - \\
\hline Total pathogenic & 2 & 0.3 & 0 & 0 & & \\
\hline Total intronic VUS & 13 & 2.1 & 2 & 1.8 & & \\
\hline Total coding VUS & 5 & 0.8 & 0 & 0 & & \\
\hline
\end{tabular}

het heterozygous, hom homozygous, MAF minor allele frequency

${ }^{\text {a }}$ No combinations of bi-allelic pathogenic mutations were found. Many patients carried multiple variants, of which the variants found in combination with variants found in $3 / 303$ patients or less are mentioned here: c.56G $>\mathrm{A}$ and c.64G $>\mathrm{A}, \mathrm{c} .312 \mathrm{C}>\mathrm{T}$ and c. $1544 \mathrm{C}>\mathrm{T}, \mathrm{c} .36+75 \mathrm{C}>\mathrm{G}$ and c. $-127 \mathrm{C}>\mathrm{T}(2 / 2$ carriers $)$, c. $388+56 \mathrm{G}>\mathrm{A}$ and c. $-127 \mathrm{C}>\mathrm{T}(1 / 2), \mathrm{c} .925 \mathrm{C}>\mathrm{T}$ and c.504 $+35 \mathrm{G}>\mathrm{A}$ and c. $1477-40 \mathrm{G}>\mathrm{C}(1 / 3), \mathrm{c} .998-27 \mathrm{G}>\mathrm{A}$ and c. $504+35 \mathrm{G}>\mathrm{A}$ and c. $1477-40 \mathrm{G}>\mathrm{C}(1 / 3)$, c. $1186+46 \mathrm{G}>\mathrm{A}$ and c. $-127 \mathrm{C}>\mathrm{T}$ and c. $1014 \mathrm{G}>\mathrm{C}$

b Minor alleles of these two variants were detected in the same patients, indicating strong LD

c The 303 consecutively accrued incident BC patients selected from the ORIGO cohort for BC diagnosis before the age of 55 years, among whom 56 had multiple primary breast tumors (bilateral or ipsilateral). This group was slightly enriched for patients with multiple breast tumors, by selection of the last 10 patients for this characteristic

d A likely classification based on data and MAF from literature and dbSNP. Two dbSNP variants were also genotyped in this study, but were not detected by sequencing in these 303 samples, namely c.536A $>\mathrm{G}$ (not present in these 303 samples, rs 34612342 ) and c.37-2487G $>\mathrm{T}$ (outside the sequenced region, rs3219476). The genotyped variant c.1214C $>$ T, p.Arg309Cys, was not detected in the 303 samples and also not present in dbSNP

compared to the patients with a single breast tumor (Table 1).

Case-control study by genotyping selected variants

We selected five candidate variants and four tagging SNPs in $M U T Y H$ for further genotyping in the entire group, totaling the 1,469 incident BC patients (ORIGO cohort), 1,666 controls, and 471 subjects at increased risk for carrying $B R C A 1 / 2$ mutations (BRCAx). The candidate variants included the three common Dutch founder mutations
(p.Tyr179Cys, p.Gly396Asp, and p.Pro405Leu), and two non-synonymous variants detected more than once in the 303 sequenced patients [p.Arg309Cys (VUS) and p.Ser515Phe (rare polymorphism)]. The four tagging SNPs were selected from the HapMap database (http:// hapmap.ncbi.nlm.nih.gov/), as representative for $M U T Y H$ polymorphisms with minor allele frequencies (MAF) of 5\% or higher. Of these four tagging SNPs, three were in the area covered by direct sequencing (p.Val22Met, c. $504+35 \mathrm{G}>\mathrm{A}$, and p.Gln338His) and one was located outside this area, in intron 1 (c.37-2487G $>$ T) (Table 1). No deviations from HWE were observed in any group. 


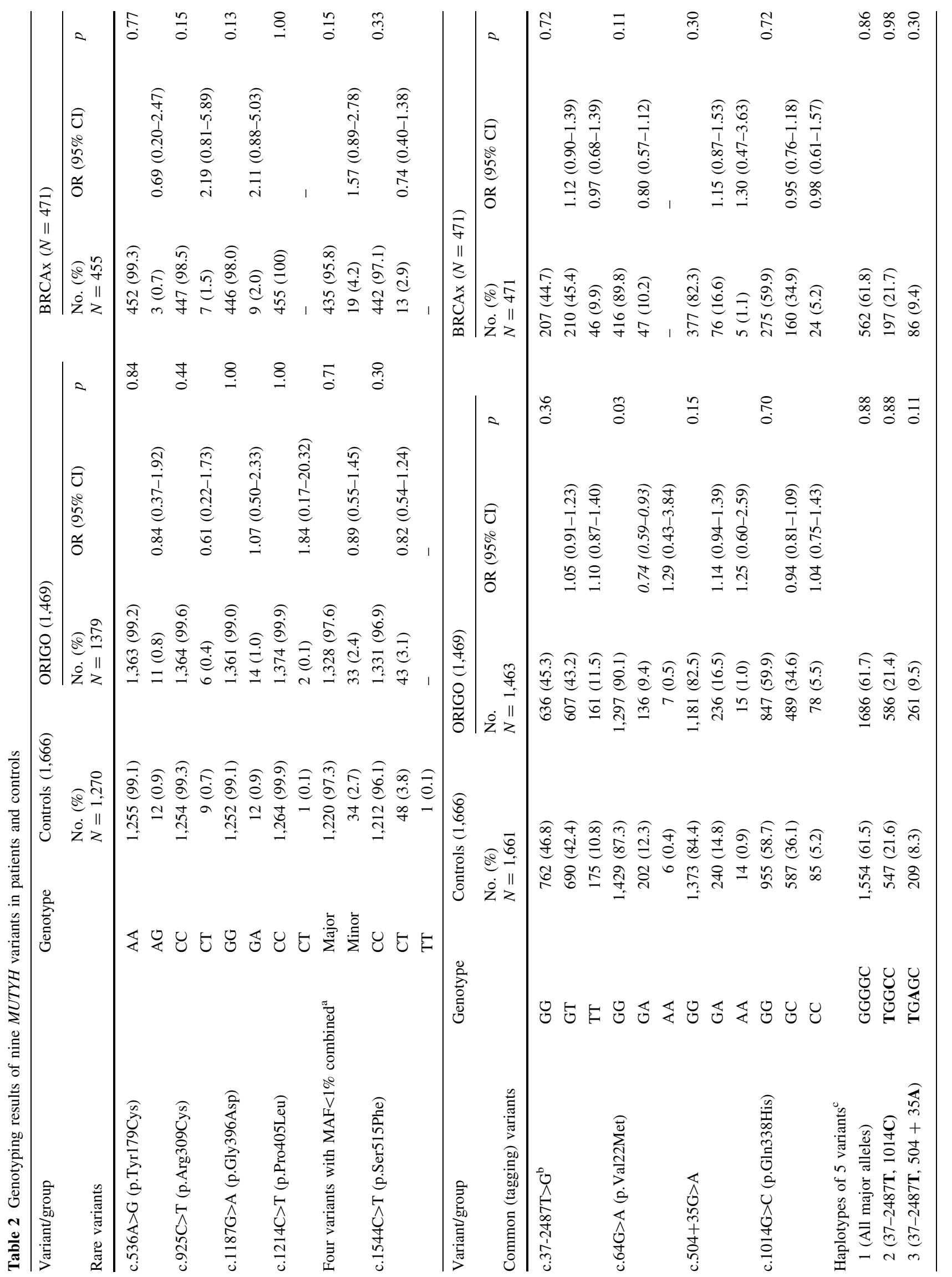




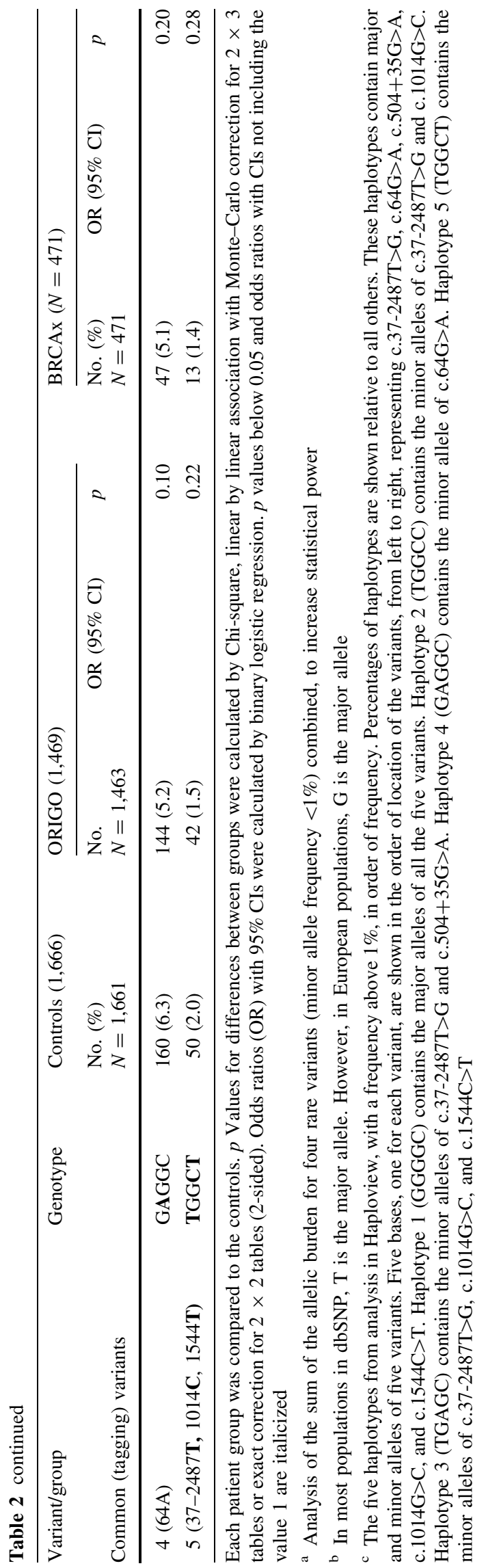

In the incident BC patient, BRCAx and control groups, no bi-allelic combinations of pathogenic mutations were detected. One control sample was homozygous (bi-allelic) for the rare polymorphism, p.Ser515Phe. Heterozygotes for the polymorphism, p.Val22Met, occurred less frequently among the incident $\mathrm{BC}$ patients $(9.4 \%$, OR $0.74,95 \%$ confidence interval (CI) $0.59-0.93, p=0.03$ ), as compared to the controls $(12.3 \%)$, suggesting a protective effect of the minor allele. A similar trend observed in the BRCAx group (Table 2). The frequency of the pathogenic variant, p.Gly396Asp, was approximately twice as high in the BRCAx subjects $(2.0 \%$, OR 2.11, 95\% CI 0.88-5.03, $p=0.13)$ as in the controls $(0.9 \%)$, and the incident $\mathrm{BC}$ patients $(1.0 \%)$. Likewise, the frequency of the VUS p.Arg309Cys was twice as high in the BRCAx subjects (1.5\%, OR 2.19, 95\% CI 0.81-5.89, $p=0.15)$ as in the controls $(0.7 \%)$, and the incident BC patients $(0.4 \%$, Table 2). The increased frequencies of p.Gly396Asp and p.Arg309Cys among BRCAx subjects might suggest an enrichment of $M U T Y H$ variants in this familial group, analogous to what was found for CHEK2 [18].

On the whole, our study showed no significantly increased BC risk associated with MUTYH variants. However, for two variants, p.Gly396Asp and p.Arg309Cys, a doubled frequency of heterozygous carriers was found in the BRCAx group, compared to the incident BC group and controls, resulting in a non-significant OR of 2.1-2.2 (Table 3). Interestingly, in a study among Sephardi Jews of North African descent (389 cases and 541 controls), p.Gly396Asp heterozygotes were found to be significantly increased in BC patients $(6.7 \%)$ compared to controls (3.7\%) [29]. However, no significant difference was found for the p.Tyr179Cys and p.Gly396Asp mutations in a study of 691 incident BC patients and 812 controls from Canada [30]. Also, in a large case-control study in subjects from USA and Poland, no major role in $\mathrm{BC}$ was found for eight polymorphisms in six BER pathway genes, among which one variant in $O G G 1$ and one in MUTYH (c.-127C>T) [31]. For the common MUTYH variant p.Gln338His, no significant difference was found between $547 \mathrm{BC}$ patients and 287 controls [32]. Although our study reports the largest case-control study so far, the power was insufficient to detect frequency differences below a certain mutation frequency. We expect that an $\mathrm{OR}$ of 2.0 (incident $\mathrm{BC}$ patient group) or 2.6 (BRCAx group) or more has been excluded by our study, but for detection of smaller risks, larger studies are needed.

\section{Association of haplotypes}

To investigate a potential effect of untyped variants in linkage disequilibrium (LD) with the genotyped $M U T Y H$ variants, an analysis of haplotypes was performed. One block 


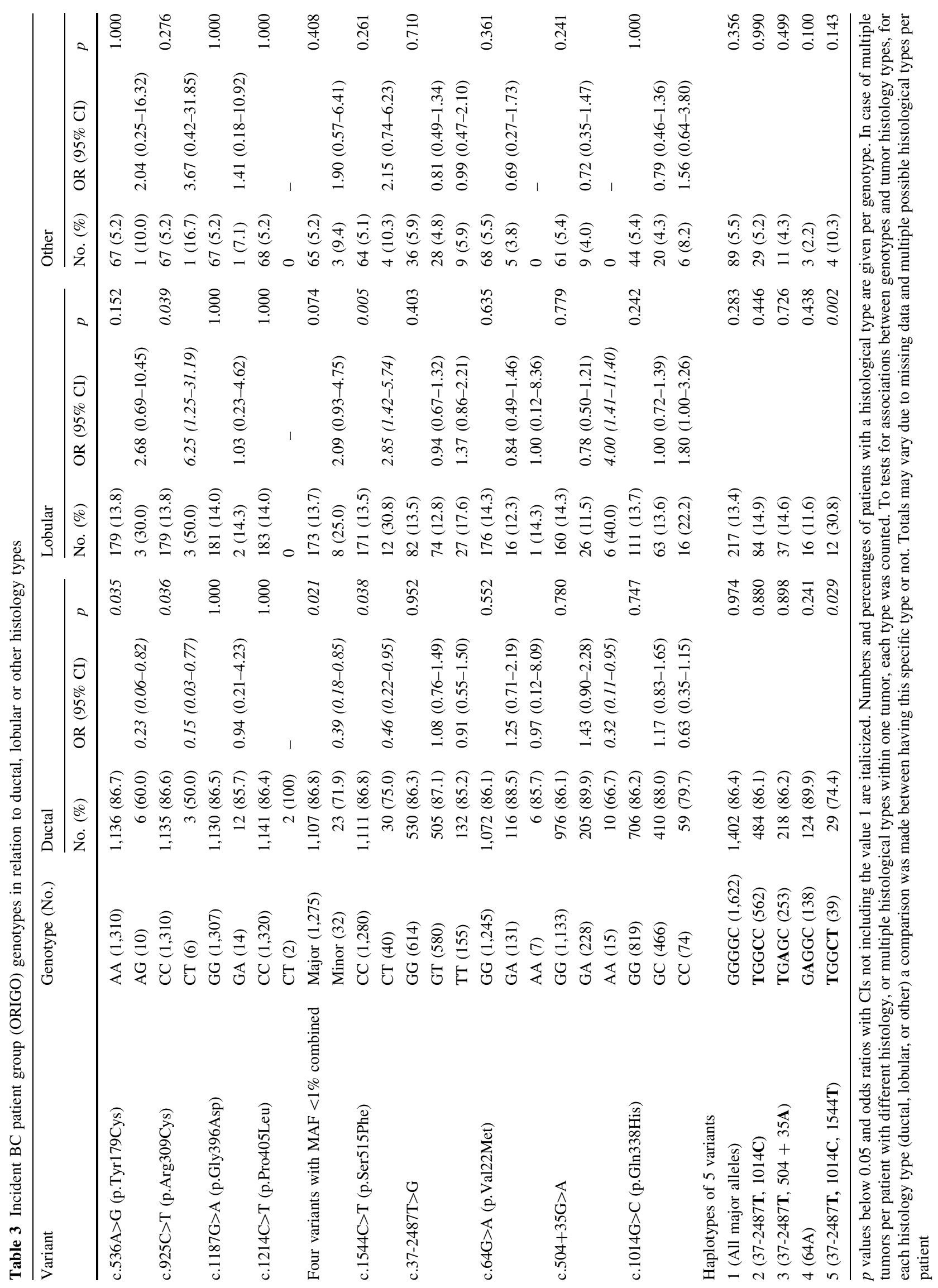


with five haplotypes with a frequency above $1 \%$ was discerned from the unphased genotypes of the four tagging polymorphisms and one candidate variant. Two haplotypes had a frequency above $20 \%$ (Tables 2,3). The fourth haplotype, carrying the minor allele of variant c.64G $>$ A (p.Val22Met) was less prevalent in the incident BC patient $(5.2 \%, p=0.10)$ and BRCAx groups $(5.1 \%, p=0.20)$ in comparison to in the controls $(6.5 \%)$. Conversely, the third haplotype, carrying the minor allele of variant c.504+ $35 \mathrm{G}>\mathrm{A}$, was more prevalent among incident $\mathrm{BC}$ patients $(9.5 \%, p=0.11)$ and BRCAx subjects $(9.4 \%, p=0.30)$ relative to controls $(8.3 \%)$. We conclude that none of the investigated MUTYH haplotypes are associated with BC, excluding the role of unknown common variants in strong LD with the genotyped variants.

Hereditary predisposition factors and tumor characteristics

Next, we evaluated a possible association of MUTYH variants with clinical features being suggestive of a hereditary predisposition. Therefore, the parameters age at diagnosis, multiple primary breast tumors in one patient (Supplementary Table S1) and BC family history (Supplementary Table S2) were analyzed in the incident BC group (ORIGO cohort). Also an association with CRC in the index patient and/or family history was tested (Supplementary Table S2). Heterozygous carriers of mutation, p.Tyr179Cys, were older at BC diagnosis (60.8 years, $n=11)$ compared to carriers of two wild-type alleles (52.7 years, $n=1,358, p=0.01$ ). Among heterozygous carriers of polymorphism, p.Ser515Phe, the frequency of patients with multiple primary breast tumors was higher (9/ $34,21 \%)$ than in carriers of two wild-type alleles (149/ $1,177,11 \%, p=0.06)$. Analyses in larger groups are necessary to confirm these trends.

To look for possible specific MUTYH effects on breast tumor characteristics, we analyzed available immunohistochemistry data and tumor histology in the incident $\mathrm{BC}$ patients (ORIGO cohort) in relation to MUTYH genotypes. No statistically significant differences were found for ER and PR (Supplementary Table S3). Heterozygotes for minor alleles of several rare variants (p.Tyr179Cys, p.Arg309Cys, and $\mathrm{p}$.Ser515Phe) showed a significantly higher frequency of lobular histology, combined with a lower frequency of ductal histology. For example, this effect was the strongest among p.Ser515Phe heterozygotes, of whom 12/40 (31\%) had one or more breast tumors with lobular histology, compared to 171/1,280 (14\%) with two wild-type, p.Ser515Phe, alleles (OR 2.9, $p=0.005$ ). Also, the homozygous genotypes of the common variants, c. $37-2487 \mathrm{~T}>\mathrm{G}$, c. $504+35 \mathrm{G}>\mathrm{A}$, and p.Gln338His, but not p.Val22Met, showed a higher frequency of lobular histology and lower frequency of ductal histology (Table 3). As we did not correct for multiple testing, we considered the significantly higher frequency of lobular histology as a trend towards association of MUTYH variants with breast tumor histology type. An association of MUTYH variants with $\mathrm{BC}$ histology has not yet been reported before. The one study investigating $\mathrm{BC}$ histology type in relation to MUTYH mutations reported no differences for ductal or other BC histology type between 30 patients heterozygous for p.Gly396Asp or p.Tyr179Cys and 359 patients homozygous wild-type for these mutations [29].

Associations with specific tumor characteristics have been found for high-risk and low risk BC genes. BRCA1related breast tumors are often "triple-negative" (i.e., showing negative immunohistochemical staining for estrogen receptor (ER), progesterone receptor (PR), and HER2/neu) [33]. Also for low risk loci, associations with breast tumor subtypes have been described [33, 34]. Lobular and ductal BCs are believed to follow different pathways in tumorigenesis [35]. It might be interesting to look for associations of MUTYH variants, specifically in a large group of lobular BC patients.

\section{Conclusions}

Our results do not confirm the data from two earlier studies which suggested a significantly increased BC risk among carriers of bi-allelic MUTYH mutations [4, 6]. No bi-allelic carriers of pathogenic MUTYH mutations were found in two large groups of $\mathrm{BC}$ patients. Also an effect of monoallelic MUTYH mutations as earlier suggested could not be significantly confirmed [7, 29]. Although our study is the largest case-control study for MUTYH variants and $\mathrm{BC}$ to date, the power is expected to be insufficient for ORs below 2.0. Several trends towards association were observed between different $M U T Y H$ variants and clinical parameters, such as the BRCAx phenotype and lobular type of BC histology, which might be interesting for future studies.

Acknowledgments We thank all the study participants, clinicians, researchers, and support staff for their contribution to this study. We are grateful to the participants in the ORIGO study workgroup: Rob A. E. M. Tollenaar, Elly M. M. Krol-Warmerdam (Department of Surgery, LUMC), Jan Molenaar (Finance Directorate, LUMC), Maaike P. G. Vreeswijk, Karin H. G. Kroeze-Jansema (Department of Human Genetics, LUMC), Pauline M. Wijers-Koster (Department of Pathology, LUMC), Cathrien E. Jacobi (Department of Medical Decision Making, LUMC), and Jannet C. Blom and Jan G. M. Klijn (Department of Medical Oncology, Family Cancer Clinic, Erasmus MC-Daniel den Hoed Cancer Center). We also thank Janneke M. Weiss (Department of Clinical Genetics, LUMC, currently VU University Medical Center) for supervision and design of the genetic tests, Ronald van Eijk (Department of Pathology, LUMC) for technical assistance, Peter de Knijff (Department of Human Genetics, LUMC) for providing part of the control samples, Twiggy C. T. E. F van Cronenburg and Hanny E. M. van Diemen-Homan (Department 
of Clinical Genetic, LUMC) for work on the data of the BRCAx samples and Eline Slagboom (Department of Molecular Epidemiology, LUMC) for guidance with the Sequenom tests. This study was financially supported by a Dutch Cancer Society grant KWF-UL2006-3601. The ORIGO study was supported by a Dutch Cancer Society grant KWF-UL-1997-1505.

Conflicts of interest The authors declare that there are no conflicts of interest.

Open Access This article is distributed under the terms of the Creative Commons Attribution License which permits any use, distribution, and reproduction in any medium, provided the original author(s) and the source are credited.

\section{References}

1. Cheadle JP, Sampson JR (2007) MUTYH-associated polyposisfrom defect in base excision repair to clinical genetic testing. DNA Repair (Amst) 6:274-279

2. Jones S, Emmerson P, Maynard J et al (2002) Biallelic germline mutations in $\mathrm{MYH}$ predispose to multiple colorectal adenoma and somatic G:C $\rightarrow$ T:A mutations. Hum Mol Genet 11:2961-2967

3. Jones S, Lambert S, Williams GT, Best JM, Sampson JR, Cheadle JP (2004) Increased frequency of the k-ras G12C mutation in MYH polyposis colorectal adenomas. Br J Cancer 90:1591-1593

4. Nielsen M, Franken PF, Reinards TH et al (2005) Multiplicity in polyp count and extracolonic manifestations in 40 Dutch patients with MYH associated polyposis coli (MAP). J Med Genet 42:e54

5. Theodoratou E, Campbell H, Tenesa A et al (2010) A large-scale meta-analysis to refine colorectal cancer risk estimates associated with MUTYH variants. Br J Cancer 103:1875-1884

6. Vogt S, Jones N, Christian D et al (2009) Expanded extracolonic tumor spectrum in MUTYH-associated polyposis. Gastroenterology 137:1976-1985

7. Wasielewski M, Out AA, Vermeulen J et al (2010) Increased MUTYH mutation frequency among Dutch families with breast cancer and colorectal cancer. Breast Cancer Res Treat 124:635-641

8. Wang X, Szabo C, Qian C et al (2008) Mutational analysis of thirty-two double-strand DNA break repair genes in breast and pancreatic cancers. Cancer Res 68:971-975

9. Li D, Zhang W, Zhu J et al (2001) Oxidative DNA damage and 8-hydroxy-2-deoxyguanosine DNA glycosylase/apurinic lyase in human breast cancer. Mol Carcinog 31:214-223

10. Soliman AS, Vulimiri SV, Kleiner HE et al (2004) High levels of oxidative DNA damage in lymphocyte DNA of premenopausal breast cancer patients from Egypt. Int $\mathrm{J}$ Environ Health Res 14:121-134

11. Dziaman T, Huzarski T, Gackowski D et al (2009) Elevated level of 8-oxo-7,8-dihydro-2'-deoxyguanosine in leukocytes of BRCA1 mutation carriers compared to healthy controls. Int $\mathrm{J}$ Cancer 125:2209-2213

12. Le Page F, Randrianarison V, Marot D et al (2000) BRCA1 and BRCA2 are necessary for the transcription-coupled repair of the oxidative 8-oxoguanine lesion in human cells. Cancer Res 60: $5548-5552$

13. Furuuchi K, Tada M, Yamada $\mathrm{H}$ et al (2000) Somatic mutations of the APC gene in primary breast cancers. Am J Pathol 156: 1997-2005

14. Sieber OM, Howarth KM, Thirlwell C et al (2004) Myh deficiency enhances intestinal tumorigenesis in multiple intestinal neoplasia (ApcMin/+) mice. Cancer Res 64:8876-8881
15. Radpour R, Fan AX, Kohler C, Holzgreve W, Zhong XY (2009) Current understanding of mitochondrial DNA in breast cancer. Breast J 15:505-509

16. Wasielewski M, Riaz M, Vermeulen J et al (2009) Association of rare MSH6 variants with familial breast cancer. Breast Cancer Res Treat 123:315-320

17. Didraga MA, van Beers EH, Joosse SA et al (2011) A nonBRCA $1 / 2$ hereditary breast cancer sub-group defined by aCGH profiling of genetically related patients. Breast Cancer Res Treat 130:425-436

18. Meijers-Heijboer H, van den Ouweland A, Klijn J et al (2002) Low-penetrance susceptibility to breast cancer due to CHEK2(*)1100delC in noncarriers of BRCA1 or BRCA2 mutations. Nat Genet 31:55-59

19. Huijts PE, Vreeswijk MP, Kroeze-Jansema KH et al (2007) Clinical correlates of low-risk variants in FGFR2, TNRC9, MAP3K1, LSP1 and 8q24 in a Dutch cohort of incident breast cancer cases. Breast Cancer Res 9:R78

20. Levy-Lahad E, Friedman E (2007) Cancer risks among BRCA1 and BRCA2 mutation carriers. Br J Cancer 96:11-15

21. Out AA, van Minderhout IJ, Goeman JJ et al (2009) Deep sequencing to reveal new variants in pooled DNA samples. Hum Mutat 30:1703-1712

22. Out AA, Tops CM, Nielsen M et al (2010) Leiden Open Variation Database of the MUTYH gene. Hum Mutat 31:1205-1215

23. Barrett JC(2009) Haploview: Visualization and analysis of SNP genotype data. Cold Spring Harb Protoc 2009:pdb.ip71

24. Aretz S, Uhlhaas S, Goergens H et al (2006) MUTYH-associated polyposis: 70 of 71 patients with biallelic mutations present with an attenuated or atypical phenotype. Int J Cancer 119:807-814

25. Peterlongo P, Mitra N, Sanchez de Abajo A et al (2006) Increased frequency of disease-causing MYH mutations in colon cancer families. Carcinogenesis 27:2243-2249

26. Cleary SP, Cotterchio M, Jenkins MA et al (2009) Germline MutY human homologue mutations and colorectal cancer: a multisite case-control study. Gastroenterology 136:1251-1260

27. Halford SE, Rowan AJ, Lipton L et al (2003) Germline mutations but not somatic changes at the MYH locus contribute to the pathogenesis of unselected colorectal cancers. Am J Pathol 162:1545-1548

28. Goto M, Shinmura K, Nakabeppu Y et al (2010) Adenine DNA glycosylase activity of 14 human MutY homolog (MUTYH) variant proteins found in patients with colorectal polyposis and cancer. Hum Mutat 31:E1861-E1874

29. Rennert G, Lejbkowicz F, Cohen I, Pinchev M, Rennert HS, Barnett-Griness O (2011) MutYH mutation carriers have increased breast cancer risk. Cancer. doi:10.1002/cncr.26506

30. Beiner ME, Zhang WW, Zhang S, Gallinger S, Sun P, Narod SA (2009) Mutations of the MYH gene do not substantially contribute to the risk of breast cancer. Breast Cancer Res Treat 114:575-578

31. Zhang Y, Newcomb PA, Egan KM et al (2006) Genetic polymorphisms in base-excision repair pathway genes and risk of breast cancer. Cancer Epidemiol Biomarkers Prev 15:353-358

32. Conde J, Silva SN, Azevedo AP et al (2009) Association of common variants in mismatch repair genes and breast cancer susceptibility: a multigene study. BMC Cancer 9:344

33. Linn SC, van 't Veer LJ (2009) Clinical relevance of the triplenegative breast cancer concept: genetic basis and clinical utility of the concept. Eur J Cancer 45(Suppl 1):11-26

34. Broeks A, Schmidt MK, Sherman ME et al (2011) Low penetrance breast cancer susceptibility loci are associated with specific breast tumor subtypes: findings from the Breast Cancer Association Consortium. Hum Mol Genet 20:3289-3303

35. Bertucci F, Orsetti B, Negre V et al (2008) Lobular and ductal carcinomas of the breast have distinct genomic and expression profiles. Oncogene 27:5359-5372 